\title{
Analytical differentiation of quinolinyl- and isoquinolinyl- substituted 1-(5-fluoropentyl)-1H-indole-3-carboxylates: 5F-PB-22 and its ten isomers
}

\author{
Erina Kohyama $^{1} \cdot$ Takao Chikumoto $^{1} \cdot$ Hiroyuki Tada $^{1} \cdot$ Kiyoyuki Kitaichi $^{2} \cdot$ \\ Tetsuro Ito ${ }^{1,2}$
}

Received: 20 June 2016/ Accepted: 27 July 2016/Published online: 12 August 2016

(C) Japanese Association of Forensic Toxicology and Springer Japan 2016

\begin{abstract}
Differentiation among regioisomers of synthetic cannabinoids in forensic drug analysis is a crucial issue, since all isomers are not regulated by law. New equivalent analogs obtained via minor modification of their preexisting molecules keep on emerging. Isomers formed via substitutional exchange are also a cause for concern. This study is focused on the isomeric molecules that stem from minor modifications of 5F-PB-22. The analytical properties of these molecules and methods of differentiation are reported. Scan mode analysis using gas chromatographyelectron ionization-mass spectrometry (GC-EI-MS) was performed using the authentic 5F-PB-22 standard, five regioisomeric quinolinyl ester indoles, and five regioisomeric isoquinolinyl ester indoles. Because it was not possible to separate 5F-PB-22 from the 5-hydroxyquinoline isomer using GC and all analytes showed similar EI mass spectra, liquid chromatography (LC)-tandem mass spectrometry analysis was performed. Using LC, a successful separation of 5F-PB-22 from all isomers could be achieved. Based on the electrospray ionization-mass spectra, the protonated molecular ion at $\mathrm{m} / \mathrm{z}, 377.2$ was selected as the
\end{abstract}

Electronic supplementary material The online version of this article (doi:10.1007/s11419-016-0334-9) contains supplementary material, which is available to authorized users.

\footnotetext{
Erina Kohyama

koyama-erina@pref.gifu.lg.jp

$\triangle$ Tetsuro Ito

ito-tetsuro@pref.gifu.lg.jp; teito@gifu-pu.ac.jp

1 Gifu Prefectural Research Institute for Health and Environmental Sciences, 1-1 Naka-fudogaoka, Kakamigahara, Gifu 504-0838, Japan

2 Gifu Pharmaceutical University, 1-25-4 Daigaku-nishi, Gifu 501-1196, Japan
}

precursor ion for the regioisomeric and structural isomeric differentiation. Collision-induced dissociation provides relative intensity differences in the product ions among the isomers, enabling mass spectrometric differentiation of the isomers. To our knowledge, this is the first report on mass spectrometric differentiation of 5F-PB-22 and its ten isomers.

Keywords Synthetic cannabinoids · Quinolinyl- and isoquinolinyl-substituted regioisomers of 5F-PB-22 - Gas chromatography-electron ionization-mass spectrometry . Liquid chromatography-tandem mass spectrometry . Regioisomer differentiation

\section{Introduction}

An increasing number of new psychoactive designer drugs have emerged worldwide during the last decade [1]. Synthetic cannabinoids (SCs) constitute a major share of drug abuse in Japan as well as in European countries [2-5]. A survey of designer drugs available in the Japanese market was conducted under the leadership of the National Institute of Health Sciences (Tokyo). The identification of known SCs and the structural determination of newly emergent SCs were achieved using a combination of technologies such as hyphenated chromatographic procedures [gas chromatography-tandem mass spectrometry (GC-MS-MS) and liquid chromatography (LC)-MS-MS] and nuclear magnetic resonance spectroscopy [6-10].

Since new structurally modified analogs have been continuously emerging in the market, avoiding/reducing expansion of such illegal species is a never-ending challenge for governmental authorities, and the "cat-andmouse game" between regulators and drug manufacturers 
is ongoing. For effective control of these new psychoactive substances, several legislative approaches have been implemented at the national level. The Ministry of Health, Labour and Welfare of Japan amended the Pharmaceutical Affairs Law in 2006 to implement a new regulatory category, called "Designated Substances (DSs)". The most effective deterrent, a comprehensive regulatory system (generic scheduling) for naphthoylindole-type SCs and synthetic cathinones, was introduced to DSs in 2013. As of April 2016, 2343 compounds are controlled as DSs. The number of vendors on the street and the Internet has been reduced after the repeated expansion of DSs. On the contrary, such government control of designer SCs may ironically result in the continuing production of new designer drugs and an expansion of structurally related analogs in the clandestine market.

SCs bear the agonistic property for both cannabinoid receptors $\left(\mathrm{CB}_{1}\right.$ and $\left.\mathrm{CB}_{2}\right)$, with various affinities and selectivities. The potential pharmacological activity on the $\mathrm{CB}_{1}$ receptors is responsible for the psychological effect and drug abuse [11, 12]. In the indole class SCs, indole-3-carboxylic acid ester derivatives with variation of the functional groups on the indole core constitute one of the representative SC groups in the DS list in Japan. The indole-3-carboxylates substituted by the 8-quinolinyl moiety (e.g., PB-22, 5F-PB22, BB-22, FUB-PB-22) and the 1-naphthoyl moiety (e.g., FDU-PB-22) constitute the majority in this class (Fig. 1). 5FPB-22 (also known as 5F-QUPIC) has been controlled as DS in 2013, and then has been re-categorized from DS to a narcotic in 2014 in Japan. Despite strict control under the Narcotics and Psychotropics Control Law, continuous survey of designer drugs in illegal markets by several prefectural governments, including Gifu, resulted in the ongoing detection of 5F-PB-22 in 2015 [13].

The next wave of SCs may include the structural variations resulting from positional isomerism. The fundamental studies on the positional isomer differentiation have

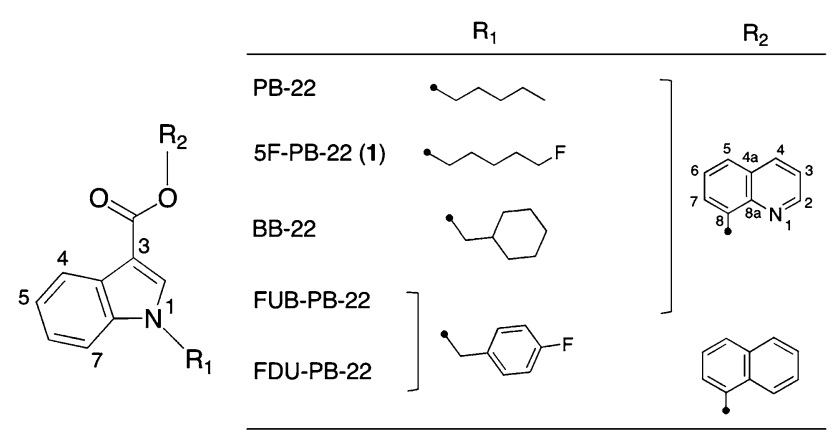

Fig. 1 Chemical structures of synthetic cannabinoids having an indole core with 3-carboxylic acid ester only been performed using 1-alkyl-3-acylindoles, including JWH-081 and JWH-018, under the prospect of their future abuse [14-21]. However, there are no reports about esteror amide-type SCs. We focused on 5F-PB-22, one of the ester-type SCs, and investigated the positional isomer differentiation of 5F-PB-22 involving five hydroxyquinoline isomers and five hydroxyisoquinoline isomers using GCMS and LC-MS-MS.

\section{Materials and methods}

\section{Reagents and chemicals}

The structures of the 11 quinolinyl- and isoquinolinylsubstituted 1-(5-fluoropentyl)- $1 H$-indole-3-carboxylates used in this study are shown in Figs. 1 and 2. Quinolin-8-yl 1-(5-fluoropentyl)-1 $H$-indole-3-carboxylate (5F-PB-22: 1); its five hydroxyquinoline isomers [5F-PB-22 7-hydroxyquinoline isomer (7Q isomer, 2), 5F-PB-22 6-hydroxyquinoline isomer (6Q isomer, 3), 5F-PB-22 5-hydroxyquinoline isomer (5Q isomer, 4), 5F-PB-22 4-hydroxyquinoline isomer (4Q isomer, 5), 5F-PB-22 3-hydroxyquinoline isomer (3Q isomer, 6)]; and its five hydroxyisoquinoline isomers [5F-PB-22 8-hydroxyisoquinoline isomer (8IQ isomer, 7), 5F-PB-22 7-hydroxyisoquinoline isomer (7IQ isomer, 8), 5F-PB-22 6-hydroxyisoquinoline isomer (6IQ isomer, 9), 5F-PB-22 5-hydroxyisoquinoline isomer (5IQ isomer, 10), and 5FPB-22 4-hydroxyisoquinoline isomer (4IQ isomer, 11)] were purchased from Cayman Chemical (Ann Arbor, MI USA). The stock standard solutions of these compounds were prepared at a concentration of $1 \mathrm{mg} / \mathrm{mL}$ (in acetonitrile) and were stored at $-20{ }^{\circ} \mathrm{C}$ until analysis. These solutions were diluted in acetonitrile to appropriate concentrations prior to analysis. All other chemicals and solvents were of analytical reagent grade or LC-MS grade. Twelve herbal products (GP-2015-01 to GP-2015-12) were purchased via the Internet in March 2015 in Japan. All the products contained approximately 3-4 g of mixed dried plants.

\section{Preparation of sample solution}

The herbal-type product ( $30 \mathrm{mg}$ ) was crushed to a powder and extracted with $3 \mathrm{~mL}$ of methanol or acetonitrile under ultrasonication for $10 \mathrm{~min}$. After centrifugation $(5 \mathrm{~min}$, $3000 \mathrm{rpm}$ ), the supernatant solution was passed through a membrane filter (Millex-LH $0.45 \mu \mathrm{m}$ filter unit; Merck Millipore, Darmstadt, Germany). Moreover, if necessary, the solution was diluted with methanol or acetonitrile to a suitable concentration before instrumental analysis. 
<smiles></smiles>

$7 \mathrm{Q}$ isomer (2)

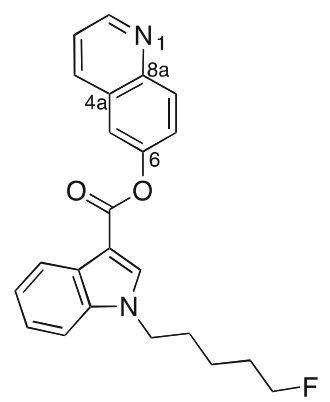

$6 \mathrm{Q}$ isomer (3)

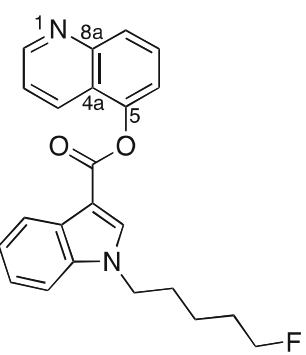

$5 \mathrm{Q}$ isomer (4)

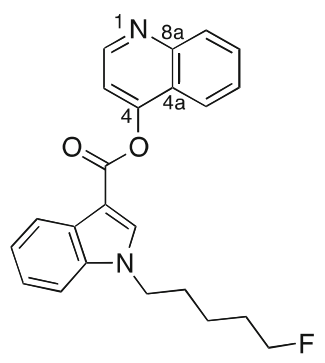

$4 \mathrm{Q}$ isomer (5)

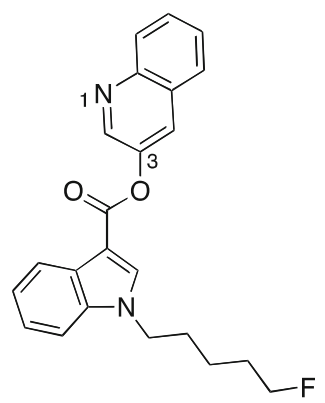

$3 Q$ isomer (6)

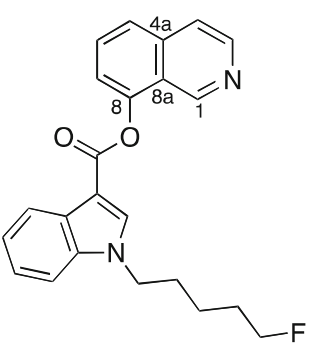

8IQ isomer (7)

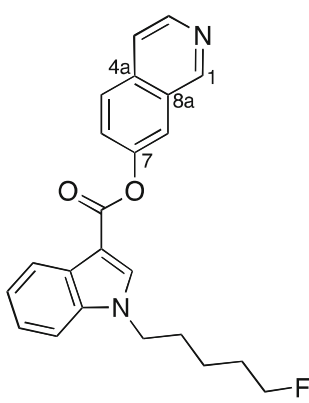

7IQ isomer (8)

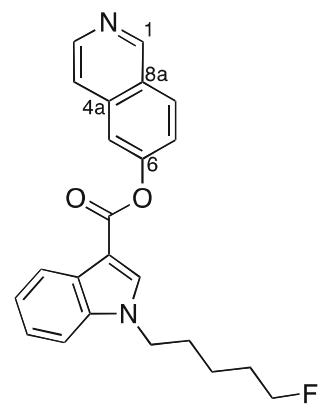

$6 \mathrm{IQ}$ isomer (9)

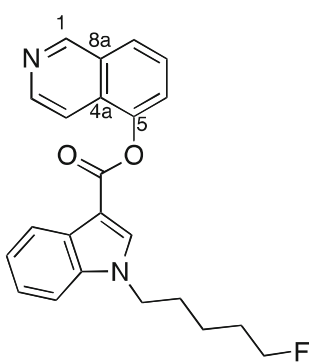

$5 \mathrm{IQ}$ isomer (10)

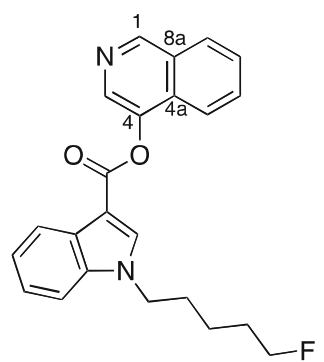

$4 I Q$ isomer (11)
Fig. 2 Chemical structures of ten isomers of 5F-PB-22 (1). 2, 5F-PB22 7-hydroxyquinoline isomer (7Q isomer); 3, 5F-PB-22 6-hydroxyquinoline isomer (6Q isomer); 4, 5F-PB-22 5-hydroxyquinoline isomer (5Q isomer); 5, 5F-PB-22 4-hydroxyquinoline isomer (4Q isomer); 6, 5F-PB-22 3-hydroxyquinoline isomer (3Q isomer); 7, 5F-

\section{Analytical methods}

GC-MS analysis was performed using a TRACE 1310 GC and ISQ LT (Thermo Fisher Scientific, Waltham, MA, USA). Samples were separated using a DB-5MS capillary column (30 $\mathrm{m} \times 0.25 \mathrm{~mm}$ i.d.; $0.25 \mu \mathrm{m}$ film thickness; Agilent Technologies, Santa Clara, CA, USA) with helium as the carrier gas, at a constant flow rate of $1.0 \mathrm{~mL} / \mathrm{min}$. The oven temperature was programmed as follows: held at $50{ }^{\circ} \mathrm{C}$ for $1 \mathrm{~min}$, linearly ramped at $10^{\circ} \mathrm{C} / \mathrm{min}$ to $310^{\circ} \mathrm{C}$, and held at $310^{\circ} \mathrm{C}$ for $12 \mathrm{~min}$. The injector temperature was set to $250{ }^{\circ} \mathrm{C}$, and the injection volume was $1 \mu \mathrm{L}$ (splitless mode). The GC interface and ion-source temperature were maintained at 280 and $250{ }^{\circ} \mathrm{C}$, respectively. Ionization was performed in the electron ionization (EI) mode at $70 \mathrm{eV}$. A mass spectral library search was performed using the Searchable Mass Spectral Library Version 2.2 downloaded from the provider's website [22].

GC-MS-MS was performed using an Agilent 7890A/7000 GC/MS Triple Quad (Agilent Technologies). The positive EI mode was employed. Samples were introduced via a DB5MS + DG capillary column $(30 \mathrm{~m} \times 0.25 \mathrm{~mm}$ i.d.; $0.25 \mu \mathrm{m}$ film thickness with $10 \mathrm{~m}$ DuraGuard; Agilent Technologies).
PB-22 8-hydroxyisoquinoline isomer (8IQ isomer); 8, 5F-PB-22 7-hydroxyisoquinoline isomer (7IQ isomer); 9, 5F-PB-22 6-hydroxyisoquinoline isomer (6IQ isomer); 10, 5F-PB-22 5-hydroxyisoquinoline isomer (5IQ isomer); and 11, 5F-PB-22 4-hydroxyisoquinoline isomer (4IQ isomer)

The analytical conditions were similar to those in the GC-MS analysis. Product ion spectra were obtained using $\mathrm{N}_{2}$ as the collision gas $(1.5 \mathrm{~mL} / \mathrm{min})$ by varying the collision energy (CE) from 5 to $35 \mathrm{~V}$, in units of $10 \mathrm{~V}$.

LC equipped with a photodiode array (PDA) detector analysis was performed on an Agilent Technologies liquid chromatograph (1100 series). Chromatographic separation was carried out on an Atlantis T3 column $(150 \times 4.6 \mathrm{~mm}$ i.d., particle size $5 \mu \mathrm{m}$; Waters, Milford, MA, USA). The mobile phase comprised (A) $0.1 \%$ formic acid in water and (B) $0.1 \%$ formic acid in acetonitrile. The column was maintained at $40{ }^{\circ} \mathrm{C}$, and the gradient program was as follows: linear gradient of $\mathrm{B}$ from 5 to $90 \%$ from 0 to $45 \mathrm{~min}$ and isocratic elution of $90 \% \mathrm{~B}$ from 45 to $60 \mathrm{~min}$. The flow rate and the injection volume were $1.0 \mathrm{~mL} / \mathrm{min}$ and $10 \mu \mathrm{L}$, respectively.

LC-MS analysis was performed on an Agilent 1100 Series LC/MSD-equipped with electrospray ionization (ESI) and an Atlantis T3 column $(150 \times 2.1 \mathrm{~mm}$ i.d., particle size $5 \mu \mathrm{m}$; Waters). The mobile phase and column temperature were similar to those in LC-PDA. The flow rate and injection volume were $0.2 \mathrm{~mL} / \mathrm{min}$ and $2 \mu \mathrm{L}$, respectively. 
LC-MS-MS analysis was performed on a 1200 Series LC (Agilent Technologies) and a 4000 QTRAP (AB Sciex, Framingham, MA, USA) equipped with ESI setup and an Atlantis T3 column $(150 \times 2.1 \mathrm{~mm}$ i.d., particle size $5 \mu \mathrm{m}$; Waters). The mobile phase and column temperature were the same as those in LC-PDA. The flow rate was $0.2 \mathrm{~mL} / \mathrm{min}$, and the injection volume was $1 \mu \mathrm{L}$. The MS was operated in the positive mode. The ion spray voltage was $5500 \mathrm{~V}$, and the desolvation temperature was $600{ }^{\circ} \mathrm{C}$. Product ion spectra were obtained at CEs of 10, 20, 30, and $35 \mathrm{~V}$.

\section{Results and discussion}

\section{Identification of 5F-PB-22 (1) from herbal products}

During the course of the Gifu prefectural governmental program to suppress drug abuse in 2015, 12 unknown herbal-type products (GP-2015-01 to GP-2015-12) were purchased on the Internet, and comprehensive analyses were performed using GC-MS, LC-PDA, and LC-MS. Identification of the detected peaks from each analytical technique was first achieved using the available database and finally by comparison of the data obtained from an authentic standard. This resulted in the identification and quantification of a narcotic 5F-PB-22 from two herbal products, GP-2015-04 (65 mg/g) and GP-2015-05 (82 mg/ g), respectively named LAST Kou No. 1 and No. 2 [13]. LC-PDA and LC-MS analyses of the two herbal products showed a peak that had retention time (Rt), UV spectrum, and mass spectrum coincident with those of the reference standard of 5F-PB-22. The results of GC-MS analysis obtained from GP-2015-05 are shown in Fig. S1. The examination directed toward detections of the quinolinyland isoquinolinyl-substituted regioisomers of 5F-PB-22 were also conducted thoroughly by all the procedures described in the following sections. However, the authors failed to detect the isomer(s) from the authentic herbal products.

It is well known that SCs having a heat-unstable core skeleton of ( $1 H$-indole)-3-carboxylate tend to give analytical artifacts, resulting from the transesterification reaction, when they are analyzed by GC-MS in the presence of methanol or ethanol [23, 24]. The degradation and esterification occur in the injection port of the GC instrument. We also demonstrated the detection of decomposed 5F-PB-22 during the analysis of GP-2015-04 and GP-2015-05. Intense peaks corresponding to the methyl ester (methyl 1-(5-fluoropentyl)-(1H-indole)-3-carboxylate $) \quad(\mathrm{Rt}=20.91 \mathrm{~min})$, 8-hydroxyquinoline $(\mathrm{Rt}=11.51 \mathrm{~min})$ and a small peak corresponding to the intact 5F-PB-22 $(\mathrm{Rt}=31.58 \mathrm{~min})$ were observed in the total ion chromatogram in the presence of methanol (Fig. S1a). To avoid a low sensitivity for the targeted analysis of SCs with (1H-indole)-3-carboxylate, acetonitrile was adopted as the solvent for extraction of all herbal products and in the preparation of standard solutions of authentic samples. This resulted in a higher sensitivity for the target molecule, 5F-PB-22 (Fig. S1b).

\section{GC-MS and GC-MS-MS analyses}

EI mass spectra of the reference standards of 5F-PB-22 (1) and its isomers (2-11) are shown in Fig. S2. These EI mass spectra were acquired individually following sample injection using GC-MS. 5F-PB-22 (1) and all ten isomers (2-11) equally showed a small molecular ion peaks at $m / z$ 376. The ion at $m / z 232\left([\mathrm{M}-144]^{+}: \mathrm{C}_{14} \mathrm{H}_{15} \mathrm{FNO}^{+}\right)$ occurred as the base peak for each of the 11 compounds and represents the $\alpha$-cleavage of a carbonyl group, i.e., the loss of a hydroxyquinoline radical from the molecular ion to afford an $N$-1-(5-fluoropentyl)-indolylacylium ion (Fig. 3). Additionally, all the compounds showed characteristic fragmentation patterns for 3-acylindoles, resulting in fragments at $\mathrm{m} / \mathrm{z} 144$ and 116 of similar relative intensities. 5F-PB-22 (1) and its ten isomers (2-11) were not distinguishable based on their EI mass spectra. Structural isomeric differentiation was also investigated using the collision-induced dissociation (CID) of ions for the molecular ion peak $(\mathrm{m} / \mathrm{z}, 376)$ and the base peak $(\mathrm{m} / \mathrm{z}, 144)$. The product ion spectra of $m / z, 376$ for 5F-PB-22 (1) and its ten isomers (2-11) had a very low sensitivity due to an insufficient precursor ion; most molecular ions were disrupted by $\alpha$-cleavage with EI. It was estimated that the precursor ion at $m / z, 144$ was attributed to $\mathrm{C}_{9} \mathrm{H}_{6} \mathrm{NO}^{+}$, not only due to the indolylacylium ion [18-21, 25, 26], but also due to the positively charged quinolinol moiety present in 2-6 [10, 24] or the isoquinolinol moiety in $\mathbf{7 - 1 1}$. However, all the compounds (1-11) yielded identical product ion spectra of $\mathrm{m} / \mathrm{z} 144$ (Fig. S3), which suggested that the abundance of the $\mathrm{m} / \mathrm{z}, 144$ ion was due to the 3-acylindole ring rather than the hydroxy(iso)quinoline ring. The differentiation of 5F-PB-22 (1) and its ten isomers (2-11), based on the product ion spectra acquired from GC-MSMS, was unfortunately unsuccessful.

The two characteristic fragment ions at $\mathrm{m} / \mathrm{z}, 144$ $\left(\mathrm{C}_{9} \mathrm{H}_{6} \mathrm{NO}^{+}\right)$and $116\left(\mathrm{C}_{8} \mathrm{H}_{6} \mathrm{~N}^{+}\right)$arose from the elimination of olefin (5-fluoro-1-pentene) from the $\mathrm{N}$-1-(5-fluoropentyl)-indolylacylium ion and the subsequent loss of CO [25]. The olefin elimination occurred with rearrangement of an $\mathrm{H}$-atom from the $\mathrm{m} / \mathrm{z} 232$ ion bearing a positively charged $N$-atom resonance, as illustrated in Fig. 3 [20]. The reaction requires the existence of an $\mathrm{N}$-substituent with an $H$-atom at a $\beta$ position relative to the indole core. Previous reports showed supporting evidence for the elimination mechanism in the EI mass spectra from two 


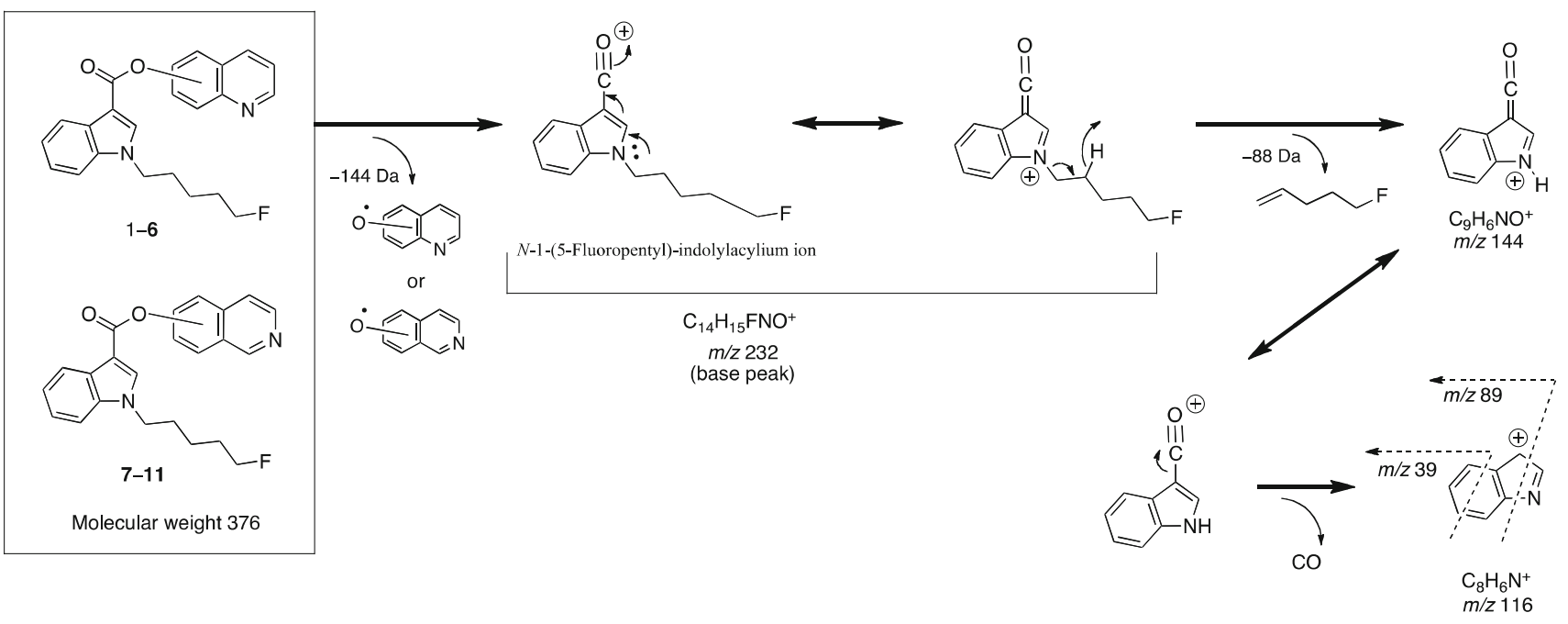

Fig. 3 Putative fragmentation scheme for 5F-PB-22 and its isomers following electron ionization

quinolin-8-yl $1 H$-indole-3-carboxylates, BB-22 [10] and FUB-PB-22 [9] (Fig. 1). The former has a cyclohexylmethyl group with a $\beta H$-atom and yields the $m / z, 144$ ion due to elimination of the substituent. The latter, substituted with a fluorobenzyl group without such an $H$-atom, did not show any $m / z, 144$ ion. This evidence further reinforces the inference that the $\mathrm{m} / \mathrm{z} 144$ ion is formed due to the indolylacylium ion alone and stems from the $\mathrm{N}$-1-(5-fluoropentyl)-indolylacylium ion at $\mathrm{m} / \mathrm{z}$ 232. The EI mass spectra of some synthetically prepared degradation products of PB-22, without a quinolinyl group, have been reported to show the intense product ion at $m / z 144$ [24], which further supports the conclusion that the $\mathrm{m} / \mathrm{z}, 144$ ion is formed from the indolylacylium ions.

\section{GC studies}

The GC Rts of 5F-PB-22 (1) and its ten isomers (2-11) are shown in Fig. 4. Under the present experimental conditions, the chromatography yielded excellent resolution between 1 and each regioisomer (2-11), except for 5Q (4) and 4Q (5) isomers. The first compound that eluted from the column was 5Q isomer $(4)(\mathrm{Rt}=31.60 \mathrm{~min})$, followed in order by $\mathbf{1}(\mathrm{Rt}=31.63 \mathrm{~min}), \mathbf{5}(\mathrm{Rt}=31.69 \mathrm{~min})$, and 5IQ isomer $(\mathbf{1 0})(\mathrm{Rt}=31.91 \mathrm{~min})$. The other compounds (2, 3, 6-9, and 11) were detected in the region $\mathrm{Rt}=32.30-33.45 \mathrm{~min} .5 \mathrm{Q}$ isomer (4) and 4Q isomer (5) were very close to $\mathbf{1}$ in retention time. Analysis of a mixture of $\mathbf{1}$ and $\mathbf{4}$ at the same concentration resulted in a single and symmetric peak (Fig. S4a). A mixture of $\mathbf{1}$ and $\mathbf{5}$ showed a split in the peak (Fig. S4b). These results show that the retention properties of $\mathbf{4}$ and $\mathbf{5}$ are extremely similar to $\mathbf{1}$ and suggest that further combined analysis using hyphenated methods must be performed for these isomers.

GC shows that six quinolinyl 1-(5-fluoropentyl)- $1 \mathrm{H}$-indole-3-carboxylates (1-6) can be grouped into two (Group A: 4, 1, and 5; Group B: 6, 3, and 2) categories according to their retention times and structural features (Fig. 4). It is interesting that compounds from group A bear a common structural feature in the quinolinyl moiety, where the $O$ atom is substituted closest to the $C$-atoms, $\mathrm{C} 4 \mathrm{a}$ or $\mathrm{C} 8 \mathrm{a}$, of the quinoline ring. These three compounds have in common the close intramolecular relationship between the two indole substituents, where a quinoline ring and indole ring situated angularly form more compact structures compared to the compounds from group B. The 5-fluoropentyl and quinolinyl groups are more crowded in these three compounds, suggesting that interactions between the two groups minimize the retention time relative to the other isomers in group B. Compounds of group B, on the other hand, also bear a common structural feature in the quinolinyl moiety, where the $O$-atom is substituted farthest from $\mathrm{C} 4 \mathrm{a}$ or $\mathrm{C} 8 \mathrm{a}$ of the quinoline ring. The quinoline ring and indole ring are positioned linearly to form an extended structure that would contribute to the similar physical properties observed in compounds from Group B. From the conformational search, performed using Pcmodel with the MMFF94 force field (Serena Software, Bloomington, IN, USA), energy-minimized structures were obtained for compounds 1-6. The results for 5F-PB-22 (1) and 7Q isomer (2) are shown in Fig. 5, and indicate a closer alignment of the quinoline ring and 5-fluoropentyl chain on the indole ring for $\mathbf{1}$ and a more extended geometry for $\mathbf{2}$. These observations support the above-mentioned arguments on the structural features of the molecules. The same 


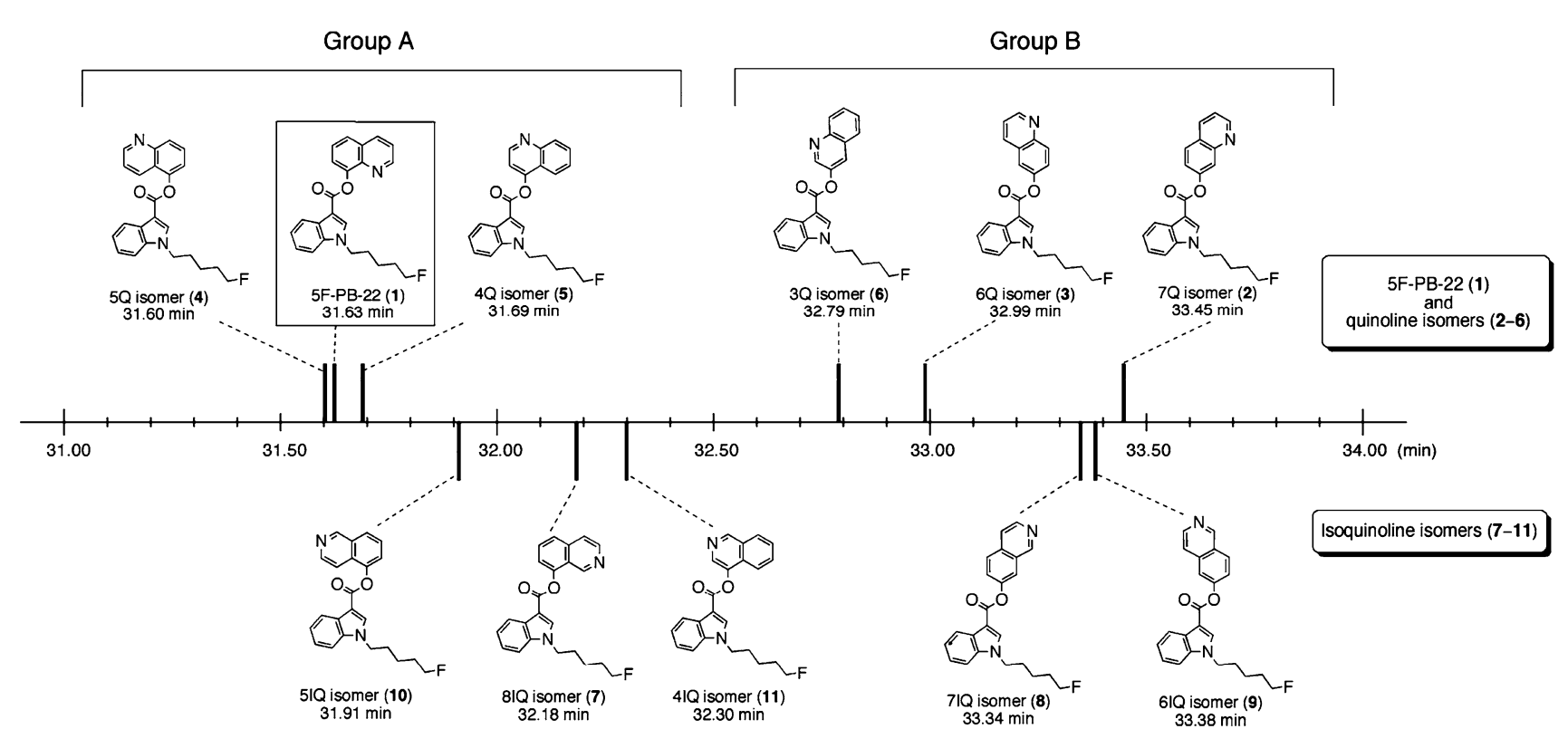

Fig. 4 Gas chromatographic retention times for 5F-PB-22 and its isomers

Fig. 5 Energy-minimized structures for $15 \mathrm{~F}-\mathrm{PB}-22$ and 2 $7 \mathrm{Q}$ isomer based on conformational search using Pcmodel with the MMFF94 force field

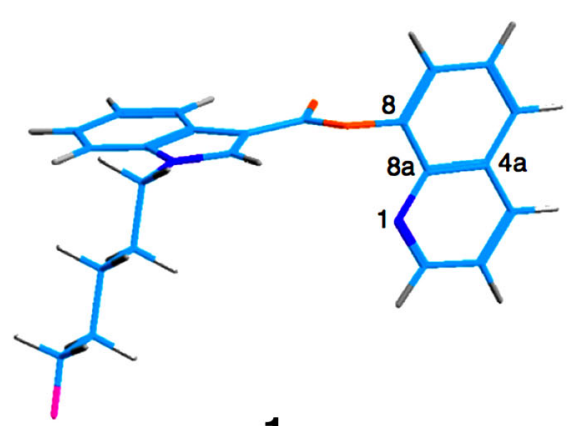

1

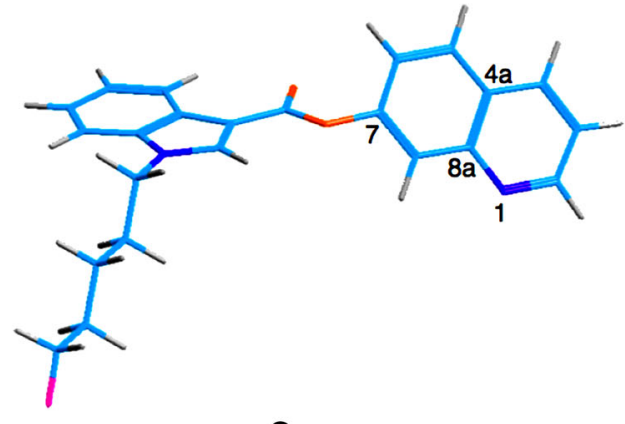

2

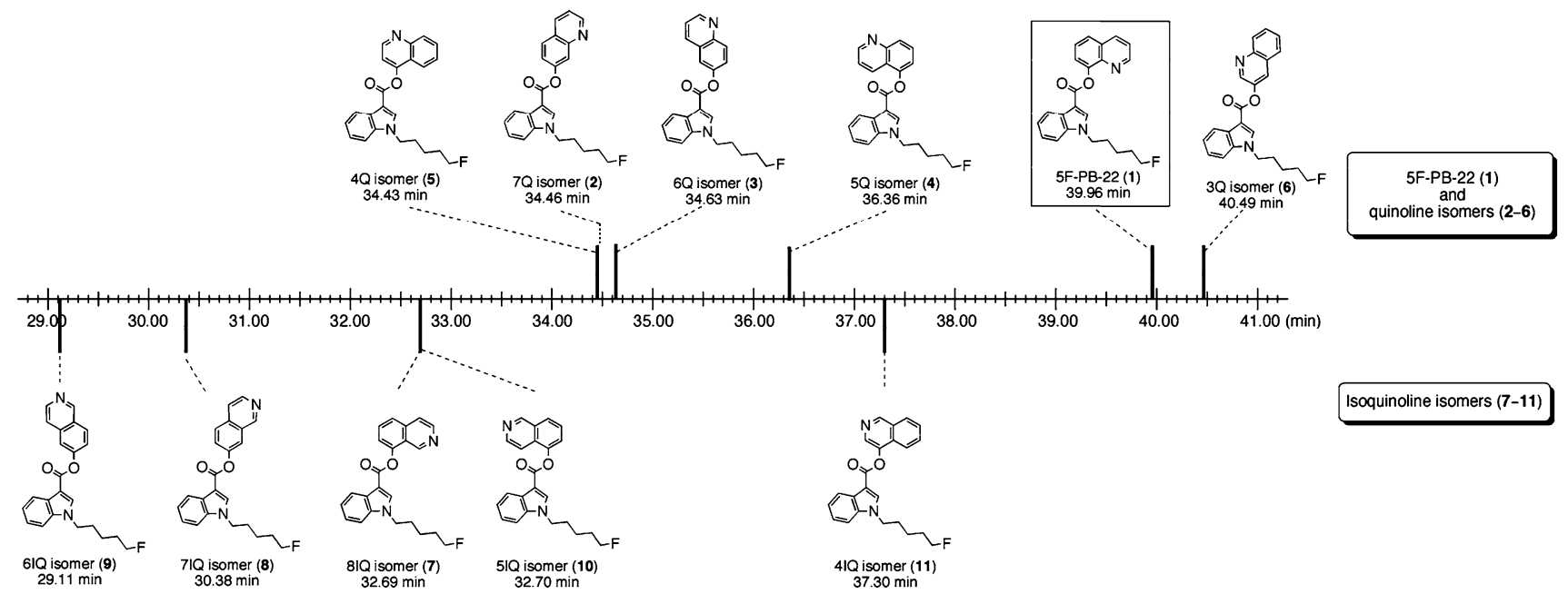

Fig. 6 Liquid chromatographic retention times for 5F-PB-22 and its isomers 
Fig. 7 Product ion spectra of precursor ion at $\mathrm{m} / \mathrm{z}, 377$ for $5 \mathrm{~F}$ PB-22 and five Q isomers at CEs of $10 \mathrm{~V}$ (left) and $20 \mathrm{~V}$ (right) on LC-MS-MS. a 5FPB-22, b 7Q isomer, c 6Q isomer, d 5Q isomer, e 4Q isomer, and $\mathbf{f} 3 \mathrm{Q}$ isomer

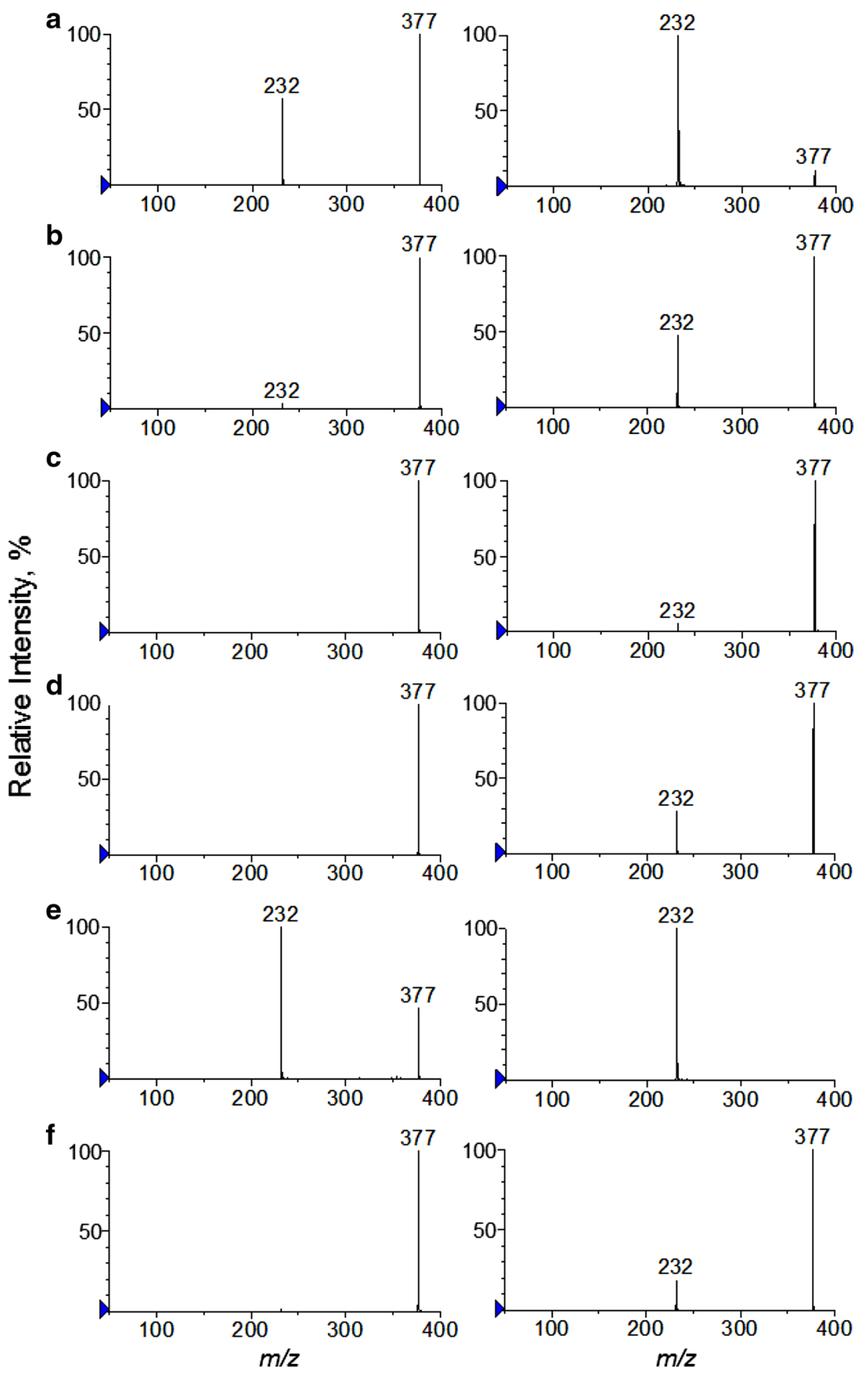

arguments apply for retention properties and the structural features of five isoquinolinyl 1 -(5-fluoropentyl)- $1 H$-indole3-carboxylates (7-11), which show a relatively shorter retention time in the case of group $\mathrm{A}(\mathbf{1 0}, \mathbf{1 1}$, and 7) with compact structural features, and an increased retention time for group B (8 and 9) with extended structural features (Fig. 4).

\section{LC-PDA and LC-MS analyses}

LC analyses, using LC-MS and LC-PDA, were employed to develop suitable procedures for the separation of $5 \mathrm{~F}$ PB-22 (1) and each of the ten isomers (2-11). The chromatogram from LC-MS showed that the peaks corresponding to 5F-PB-22 (1) and ten isomers (2-11) were in 
Table 1 Relative intensities of the product ions observed by liquid chromatography-tandem mass spectrometry

\begin{tabular}{|c|c|c|c|c|c|c|c|c|}
\hline \multirow[t]{2}{*}{ Compound } & \multicolumn{2}{|l|}{ CE $10 \mathrm{~V}$} & \multicolumn{2}{|l|}{ CE $20 \mathrm{~V}$} & \multicolumn{2}{|l|}{ CE $30 \mathrm{~V}$} & \multicolumn{2}{|l|}{ CE $35 \mathrm{~V}$} \\
\hline & $\mathrm{m} / \mathrm{z} 232$ & $\mathrm{~m} / \mathrm{z} 377$ & $\mathrm{~m} / \mathrm{z} 232$ & $\mathrm{~m} / \mathrm{z} 377$ & $\mathrm{~m} / \mathrm{z} 232$ & $\mathrm{~m} / \mathrm{z} 377$ & $\mathrm{~m} / \mathrm{z} 232$ & $\mathrm{~m} / z 377$ \\
\hline 5F-PB-22 (1) & 57.2 & 100 & 100 & 11.0 & 100 & - & 100 & - \\
\hline 7Q isomer (2) & 3.8 & 100 & 47.7 & 100 & 100 & 4.5 & 100 & - \\
\hline $6 \mathrm{Q}$ isomer $(3)$ & - & 100 & 4.9 & 100 & 87.9 & 100 & 100 & 24.4 \\
\hline $5 \mathrm{Q}$ isomer (4) & 2.8 & 100 & 28.7 & 100 & 100 & 10.6 & 100 & - \\
\hline $4 Q$ isomer $(\mathbf{5})$ & 100 & 45.9 & 100 & - & 100 & - & 100 & - \\
\hline 3Q isomer $(\mathbf{6})$ & - & 100 & 19.0 & 100 & 100 & 35.5 & 100 & - \\
\hline 8IQ isomer (7) & - & 100 & 32.1 & 100 & 100 & - & 100 & - \\
\hline 7IQ isomer $(\mathbf{8})$ & - & 100 & 2.0 & 100 & 79.6 & 100 & 100 & 38.1 \\
\hline 6IQ isomer $(\mathbf{9})$ & - & 100 & 35.1 & 100 & 100 & 3.4 & 100 & - \\
\hline $5 \mathrm{IQ}$ isomer $(\mathbf{1 0})$ & - & 100 & - & 100 & 100 & 95.0 & 100 & 26.6 \\
\hline 4IQ isomer $(\mathbf{1 1})$ & 4.1 & 100 & 63.2 & 100 & 100 & 2.6 & 100 & - \\
\hline
\end{tabular}

- Not observed; $C E$ collision energy, $Q$ hydroxyquinoline, $I Q$ hydroxyisoquinoline the region of $\mathrm{Rt}=29.11-40.49 \mathrm{~min}$ (Fig. 6). LC analysis facilitated the complete separation of $\mathbf{1}(\mathrm{Rt}=39.96 \mathrm{~min})$, $4(\mathrm{Rt}=36.36 \mathrm{~min})$, and $\mathbf{5}(\mathrm{Rt}=34.43 \mathrm{~min})$, although they overlapped during GC analysis. The compounds studied exhibited incomparable UV absorption spectra with similarity over the range of 200-350 nm (Fig. S5). All the isomeric compounds (2-11) exhibited mass spectra similar to 5F-PB-22 (1), which showed a quasi-molecular ion at $\mathrm{m} / \mathrm{z}, 377$ and a fragment ion at $\mathrm{m} / \mathrm{z} 232$, on LC-MS analysis (data not shown).

\section{LC-MS-MS analysis}

Mass spectrometric differentiation among 5F-PB-22 (1) and its ten isomers (2-11) was attempted using CID by targeting the protonated molecular ion at $\mathrm{m} / \mathrm{z}, 377.2$ as the precursor ion. The analyses were performed at CEs of 10, 20, 30, and $35 \mathrm{~V}$ using an LC-MS-MS in the ESI mode. The product ion spectra for 5F-PB-22 (1) and five Q isomers (2-7) at CEs of 10 and $20 \mathrm{~V}$ are shown in Fig. 7. The major product ion was only at $\mathrm{m} / \mathrm{z} 232$, and the other fragment ion was hardly observed. The only difference among the product ion spectra of the ten isomers was their relative intensities of the product ion at $\mathrm{m} / \mathrm{z} 232$, which was used for isomer differentiation. The relative intensities are summarized in Table 1, based on the precursor ion at $\mathrm{m} / \mathrm{z} 377$ and the product ion at $\mathrm{m} / \mathrm{z} 232$. At a CE of $10 \mathrm{~V}$, 5F-PB-22 (1) and 4Q isomer (5) produced the product ion at $\mathrm{m} / \mathrm{z} 232$ with relative intensities of 57 and $100 \%$, respectively. For nine isomers (2-4 and 6-11), other than $4 \mathrm{Q}$ isomer (5), the product ions at $\mathrm{m} / \mathrm{z} 232$ were not detected or were detected at very low relative intensities $(<10 \%)$. At a CE of $20 \mathrm{~V}, 5 \mathrm{~F}-\mathrm{PB}-22$ (1) showed ions at $\mathrm{m} / \mathrm{z} 232$ and 377 with relative intensities of 100 and $11 \%$, respectively. The precursor ion at $\mathrm{m} / \mathrm{z} 377$ was not detected at CEs of 30 and $35 \mathrm{~V}$. The relative intensities differed from isomer to isomer depending on CE. This reflects the difference in energy required for $\alpha$-cleavage that depends on the molecular structure of each isomer. Based on a relative-intensity comparison among 5F-PB-22 (1) and its five $\mathrm{Q}$ isomers (2-6), the energy required for $\boldsymbol{\alpha}$-cleavage is estimated as follows: $6 \mathrm{Q}$ isomer $>3 \mathrm{Q}$ isomer $>5 \mathrm{Q}$ isomer $>7 \mathrm{Q}$ isomer $>5 \mathrm{~F}-\mathrm{PB}-22>4 \mathrm{Q}$ isomer. As a result, isomer differentiation was possible based on the product ion spectra at several steps of CE using LC-MS-MS. It would be effective to confirm the conformity of the relative intensity with a reference standard using LC-MS-MS for preventing the misidentification of these isomers.

\section{Conclusions}

We conducted hyphenated chromatographic analysis for regioisomers of hydroxyquinolinyl ester indoles and hydroxyisoquinolinyl ester indoles of 5F-PB-22. All isomers were indistinguishable from 5F-PB-22 by EI-MS and EIMS-MS analyses coupled to a GC system, due to their superimposable spectra. SCs having a carboxy ester group at the $\mathrm{C}-3$ position of indole hardly generate a detectable ion stemming from quinolinyl- or isoquinolinyl-moiety, which renders structural differentiation difficult. However, all isomers were distinguishable from 5F-PB-22 by combining results from GC and LC separations. Although the GC system showed close retention among 5F-PB-22 (1), 5Q isomer (4), and $4 \mathrm{Q}$ isomer (5), they were completely resolved in the LC system using ODS adsorbent. The elution order in the GC system showed that the stereochemically extended isomers have a higher affinity for the capillary column. While the UV and ESI mass spectra obtained from the LC system were also not suitable for structural differentiation due to their close 
similarity, ESI-MS-MS analysis enabled the differentiation of the isomers by means of differences in relative ion intensity for the product ion at $\mathrm{m} / \mathrm{z} 232$, derived from the protonated quasi-molecular ion at $\mathrm{m} / \mathrm{z}$ 377. The recent research trend regarding the structural differentiation of SCs, supervised by Zaitsu $[14,16]$ and Clark [15, 17-20], is focused on 1-alkyl-3-acylindoles. The series of isomers in the GC system display significant fragment ions stemming from the isomeric acyl cation at equivalent masses, with some differences in relative abundance of these ions, which is the biggest advantage of using EI-MS for this purpose. On the other hand, 1-alkyl-(1H-indole)-3-carboxylates can hardly generate a detectable ion from isomeric moieties, e.g., quinolinyl ester and isoquinolinyl ester conducted in the current study, hindering the development of an analytical procedure based on EI-MS for such compounds. This problem is encountered not only in case of EI-MS-aided structural differentiation of other regioisomeric quinolinyl or isoquinolinyl ester indoles of PB-22, BB-22, and FUB-PB22, but also for indazole derivatives, e.g., 5F-NPB-22, NPB22, and FUB-NPB-22. In addition to the differentiation procedure by resolution in $\mathrm{GC}$ and $\mathrm{LC}$ retention, an alternate procedure is required for future forensic examinations. The potential procedure aimed toward tackling this challenging subject involves the utilization of product ion spectra in the ESI mode, which would contribute to the analysis of forthcoming designated substances.

Acknowledgments This work was funded by the domestically programmed grant for the regional society from the Gifu Prefectural Research Institute for Health and Environmental Sciences. The study was supported by the Health and Labour Sciences Research Grants 2015 and 2016 to K. Kitaichi (Research on Regulatory Science of Pharmaceuticals and Medical Devices, No. 27170401). A portion of this work was supported by the governmental program on a survey of designer drugs in the illegal drug market, supervised by Gifu Prefectural Government, Japan. We acknowledge the Gifu Regional Consortium on the Development of Analytical Procedures for Legal Highs operated by Gifu Pharmaceutical University and the Gifu Prefectural Research Institute for Health and Environmental Sciences for overseeing the experimental protocols.

\section{Compliance with ethical standards}

Conflict of interest There are no financial or other relations that could lead to a conflict of interest.

Ethical approval This article does not contain any studies with human participants or animals performed by any of the authors.

\section{References}

1. Underwood E (2015) A new drug war. Science 347:469-473

2. Kikura-Hanajiri R (2016) New designer drugs in Japan. In: Victor RP (ed) Neuropathology of drug addictions and substance misuse, vol 2. Academic Press, Cambridge, pp 1055-1065
3. United Nations Office on Drugs and Crime (2015) Fifteen novel synthetic cannabinoids reported to UNODC EWA since the beginning of the year. http://www.unodc.org/LSS/Announce ment/Details/d05ee4c0-c835-4055-b042-df0a15f16f49. Accessed June 2016

4. European Monitoring Centre for Drugs and Drug Addiction (2015) EMCDDA-Europol 2014 annual report on the implementation of Council Decision 2005/387/JHA, Implementation reports, Publications Office of the European Union, Luxembourg. http://www.emcdda.europa.eu/system/files/publications/1018/ TDAN15001ENN.pdf. Accessed June 2016

5. European Monitoring Centre for Drugs and Drug Addiction (2015) European drug report 2015: trends and developments. Publications Office of the European Union, Luxembourg. http:// www.emcdda.europa.eu/publications/edr/trends-developments/ 2015. Accessed June 2016

6. Uchiyama N, Kikura-Hanajiri R, Hakamatsuka T (2016) A phenethylamine derivative 2-(4-iodo-2,5-dimethoxyphenyl)- $\mathrm{N}$ [(3,4-methylenedioxyphenyl)methyl]ethanamine (25I-NB34MD) and a piperazine derivative 1-(3,4-difluoromethylenedioxybenzyl)piperazine (DF-MDBP), newly detected in illicit products. Forensic Toxicol 34:166-173

7. Uchiyama N, Asakawa K, Kikura-Hanajiri R, Tsutsumi T, Hakamatsuka T (2015) A new pyrazole-carboxamide type synthetic cannabinoid AB-CHFUPYCA [ $N$-(1-amino-3-methyl-1oxobutan-2-yl)-1-(cyclohexylmethyl)-3-(4-fluorophenyl)-1 $H$ pyrazole-5-carboxamide] identified in illegal products. Forensic Toxicol 33:367-373

8. Uchiyama N, Shimokawa Y, Kikura-Hanajiri R, Demizu Y, Goda Y, Hakamatsuka T (2015) A synthetic cannabinoid FDU-NNEI, two $2 \mathrm{H}$-indazole isomers of synthetic cannabinoids AB-CHMINACA and NNEI indazole analog (MN-18), a phenethylamine derivative $\mathrm{N}$-OH-EDMA, and a cathinone derivative dimethoxy$\alpha$-PHP, newly identified in illegal products. Forensic Toxicol 33:244-259

9. Uchiyama N, Shimokawa Y, Kawamura M, Kikura-Hanajiri R, Hakamatsuka $T$ (2014) Chemical analysis of a benzofuran derivative, 2-(2-ethylaminopropyl)benzofuran (2-EAPB), eight synthetic cannabinoids, five cathinone derivatives, and five other designer drugs newly detected in illegal products. Forensic Toxicol 32:266-281

10. Uchiyama N, Matsuda S, Kawamura M, Kikura-Hanajiri R, Goda Y (2013) Two new-type cannabimimetic quinolinyl carboxylates, QUPIC and QUCHIC, two new cannabimimetic carboxamide derivatives, ADB-FUBINACA and ADBICA, and five synthetic cannabinoids detected with a thiophene derivative $\alpha$-PVT and an opioid receptor agonist AH-7921 identified in illegal products. Forensic Toxicol 31:223-240

11. Aung MM, Griffin G, Huffman JW, Wu M, Keel C, Yang B, Showalter VM, Abood ME, Martin BR (2000) Influence of the $N$ 1 alkyl chain length of cannabimimetic indoles upon $\mathrm{CB}(1)$ and $\mathrm{CB}(2)$ receptor binding. Drug Alcohol Depend 60:133-140

12. Wiley JL, Compton DR, Dai D, Lainton JA, Phillips M, Huffman JW, Martin BR (1998) Structure-activity relationships of indoleand pyrrole-derived cannabinoids. J Pharmacol Exp Ther 285:995-1004

13. Gifu Prefectural Government (2015) Media release, 11th June. http://www.pref.gifu.lg.jp/kodomo/iryo/yakuji/11224/dappou hahbu.data/kisyahappyou1506101.pdf. Accessed May 2016

14. Kusano M, Yamanaka M, Zaitsu K, Nakayama H, Nakajima J, Moriyasu T, Tsuchihashi H, Ishii A (2016) Regioisomeric differentiation of the alkyl-substituted synthetic cannabinoids JWH122 and JWH-210 by GC-EI-MS/MS. Forensic Toxicol 34:304-315

15. Abdel-Hay KM, De Ruiter J, Smith F, Alsegiani AS, ThaxtonWeissenfluh A, Clark CR (2016) GC-MS differentiation of the 
six regioisomeric dimethoxybenzoyl-1-pentylindoles: isomeric cannabinoid substances. J Pharm Biomed Anal 125:360-368

16. Kusano M, Zaitsu K, Nakayama H, Nakajima J, Hisatsune K, Moriyasu T, Matsuta S, Katagi M, Tsuchihashi H, Ishii A (2015) Positional isomer differentiation of synthetic cannabinoid JWH081 by GC-MS/MS. J Mass Spectrom 50:586-591

17. Thaxton A, Belal TS, Smith F, DeRuiter J, Abdel-Hay KM, Clark CR (2015) GC-MS studies on the six naphthoyl-substituted 1- $\mathrm{N}$ pentyl-indoles: JWH-018 and five regioisomeric equivalents. Forensic Sci Int 252:107-113

18. Abdel-Hay KM, DeRuiter J, Smith F, Belal TS, Clark CR (2015) GC-MS analysis of the regioisomeric methoxy- and methylbenzoyl-1-pentylindoles: isomeric synthetic cannabinoids. Sci Justice 55:291-298

19. DeRuiter J, Smith FT, Abdel-Hay K, Clark CR (2014) Analytical differentiation of 1-alkyl-3-acylindoles and 1-acyl-3-alkylindoles: isomeric synthetic cannabinoids. Anal Chem 86:3801-3808

20. Smith FT, DeRuiter J, Abdel-Hay K, Clark CR (2014) GC-MS and FTIR evaluation of the six benzoyl-substituted-1-pentylindoles: isomeric synthetic cannabinoids. Talanta 129:171-182

21. Shevyrin V, Melkozerov V, Nevero A, Eltsov O, Shafran Y (2013) Analytical characterization of some synthetic cannabinoids, derivatives of indole-3-carboxylic acid. Forensic Sci Int 232:1-10
22. Scientific Working Group for the Analysis of Seized Drugs (2015) http://www.swgdrug.org/. Accessed April 2015

23. Blakey K, Boyd S, Atkinson S, Wolf J, Slottje PM, Goodchild K, McGowan J (2016) Identification of the novel synthetic cannabimimetic 8-quinolinyl 4-methyl-3-(1-piperidinylsulfonyl)benzoate (QMPSB) and other designer drugs in herbal incense. Forensic Sci Int 260:40-53

24. Tsujikawa K, Yamamuro T, Kuwayama K, Kanamori T, Iwata YT, Inoue H (2014) Thermal degradation of a new synthetic cannabinoid QUPIC during analysis by gas chromatographymass spectrometry. Forensic Toxicol 32:201-207

25. Wohlfarth A, Gandhi AS, Pang S, Zhu M, Scheidweiler KB, Huestis MA (2014) Metabolism of synthetic cannabinoids PB-22 and its 5-fluoro analog, 5F-PB-22, by human hepatocyte incubation and high-resolution mass spectrometry. Anal Bioanal Chem 406:1763-1780

26. Takayama T, Suzuki M, Todoroki K, Inoue K, Min JZ, KikuraHanajiri R, Goda Y, Toyo'oka T (2014) UPLC/ESI-MS/MSbased determination of metabolism of several new illicit drugs, ADB-FUBINACA, AB-FUBINACA, AB-PINACA, QUPIC, 5FQUPIC and $\alpha$-PVT, by human liver microsome. Biomed Chromatogr 28:831-838 